\title{
The treatment of systematically transplanted gingival mesenchymal stem cells in periodontitis in mice
}

\author{
WENDONG SUN ${ }^{1}$, ZHIGUO WANG $^{2}$, QUANCHEN XU $^{1}$, HUIBIN SUN $^{1}$, \\ XIAOXUAN LIU ${ }^{1}$, JIANJUN YANG ${ }^{1}$ and RUNDAN HONG ${ }^{1}$ \\ ${ }^{1}$ Department of Stomatology, Key Lab of Oral Clinical Medicine, The Affiliated Hospital of Qingdao University, \\ College of Stomatology, Qingdao University; ${ }^{2}$ Department of Burn and Plastic Surgery, The Affiliated \\ Hospital of Qingdao University, Qingdao, Shandong 266003, P.R. China
}

Received January 17, 2018; Accepted November 11, 2018

DOI: $10.3892 /$ etm.2019.7165

\begin{abstract}
Cell-based tissue engineering is a promising alternative for periodontal regeneration as current therapies fail to reconstitute tissue damage caused by periodontitis. As newly identified postnatal stem cells, gingiva-derived mesenchymal stem cells (GMSCs) have been focused on for isolation and expansion in vitro of cells with multidifferentiation potential and immunomodulatory capacities. It has been demonstrated that systemically delivered GMSCs can home to the mandibular bone defect sites and promote bone regeneration. However, the role of transplanted GMSCs in the treatment of periodontitis has not been reported. In the present study, GMSCs were transplanted into C57BL/6J mice with periodontitis via the tail vein to investigate the contribution of GMSCs to periodontal tissue regeneration. Results demonstrated that the alveolar bone heights of mice with transplanted GMSCs were significantly increased compared with the control groups and GMSCs were detected in newly formed periodontal ligament and alveolar bone. The results of the present study implied that systemically transplanted GMSCs could home to periodontal injury sites and promote periodontal tissue regeneration.
\end{abstract}

\section{Introduction}

Periodontal diseases are bacteria-induced chronic inflammatory diseases which affect the teeth-supporting tissues and are highly prevalent worldwide (1). Periodontitis is one of the most common forms of periodontal disease and is characterized by

Correspondence to: Dr Quanchen Xu or Dr Huibin Sun, Department of Stomatology, Key Lab of Oral Clinical Medicine, The Affiliated Hospital of Qingdao University, College of Stomatology, Qingdao University, 16 Jiangsu Road, Qingdao, Shandong 266003, P.R. China

E-mail: qyfyxqc@126.com

E-mail:shb353.qindao@163.com

Key words: gingiva, mesenchymal stem cells, systematical transplantation, periodontitis, mice periodontal pocket formation, attachment loss and alveolar bone resorption. Periodontal therapy aims to achieve periodontal regeneration by reforming gingival connective tissue, cementum and alveolar bone (2). Unfortunately, due to the complex and particular etiology of periodontitis and the periodontal microenvironment, traditional treatment strategies fail to repair damage through periodontal disease completely (3). With the increasing knowledge of stem cells and tissue engineering, stem cell-based therapy is a promising candidate for periodontal regeneration and identifying the ideal seed cells which is an important part (4).

Mesenchymal stem cells (MSCs) emerged as an alternative cell source for tissue engineering due to their self-renewal ability and multi-differentiation potential (5). Bone marrow stromal cells (BMSCs) and several kinds of dental mesenchymal stem cells have been proved to promote periodontal regeneration (6). Dental mesenchymal stem cells are MSCs that are harvested from a dental body and subsidiary tissue, including the periodontal ligament (7), dental pulp (8) and dental follicle (9). However, certain drawbacks and limitations hinder the common application of these MSCs. For instance, the process of bone marrow isolation is an invasive procedure for donors and the yield of BMSCs is small. The proliferative and differentiation potential of BMSCs decrease during long-term culture and certain specific growth factors are usually needed to prolong the lifespan and differentiation ability of BMSCs (10). Dental MSCs are generally obtained from extracted teeth, but these cells are also not commonly isolated or cultured. The gingival MSCs (GMSCs) are stem cells derived from gingival tissue in recent years, which possess MSC characteristics including self-renewal, clonogenicity, multi-differentiation and expression of MSCs associated surface markers (11). GMSCs are easy to isolate and exhibit a stable phenotype, maintain a normal karyotype and telomerase activity in long-term cultures. GMSCs have been confirmed to participate in periodontal defect regeneration in animal models $(12,13)$. In the previous study, green fluorescent protein (GFP)-labeled GMSCs transplanted via the tail vein into mice with mandibular bone defects were demonstrated to home to the defect sites and promoted bone regeneration (14). However, the effect of systemically transplanted GMSCs on periodontitis in mice is still unknown. 
In the present study, GMSCs labeled with GFP were transplanted into $\mathrm{C} 57 \mathrm{BL} / 6 \mathrm{~J}$ mice with periodontitis in the second maxillary molar induced by silk thread ligation. GFP detection, morphometric and histopathological analysis were performed to evaluate the contribution of GMSCs to periodontitis.

\section{Materials and methods}

Animals. A total of 36 C57BL/6J mice (weight, 21.08 $\pm 2.14 \mathrm{~g}$ ) were obtained from the Peking University Health Science Center (Beijing, China) and kept under specific-pathogen-free conditions with controlled temperature $\left(22 \pm 2^{\circ} \mathrm{C}\right)$, humidity (60\%) and lighting (12-h light/dark cycle), and access to sterile food and water. Mice were used for experiments at the age of 8 weeks. All animal-experiment procedures described in this study were reviewed and approved by the Institutional Animal Care and Use Committee of Qingdao University, (Qingdao, China).

Isolation of GMSCs. Human gingival samples were harvested from four healthy volunteers ( 2 males and 2 females; age, 18-25 years) who underwent routine dental procedures at the Department of Stomatology, Affiliated Hospital of Medical College, Qingdao University were obtained. All procedures are approved by the clinical research ethics committee of Qingdao University and informed consent was provided. The gingival tissues were washed several times by PBS containing $400 \mu \mathrm{g} / \mathrm{ml}$ streptomycin and $400 \mathrm{U} / \mathrm{ml}$ penicillin and the tissues were incubated overnight with $\alpha$-minimum essential medium (MEM; Hyclone, Logan, Utah, USA) containing $2 \mathrm{mg} / \mathrm{ml}$ dispase (Sigma-Aldrich; Merck KGaA, Darmstadt, Germany) at $4^{\circ} \mathrm{C}$. Following the epithelial layer separation, the connective tissues were minced into fragments and digested with $2 \mathrm{mg} / \mathrm{ml}$ collagenase (Sigma-Aldrich; Merck $\mathrm{KGaA}$ ) at $37^{\circ} \mathrm{C}$ for $40 \mathrm{~min}$. The tissue explants were placed into a $25 \mathrm{~mm}^{2}$ culture flask containing $\alpha$-MEM medium with $15 \%$ fetal bovine serum (FBS; Hyclone) at $37^{\circ} \mathrm{C}$ in $5 \% \mathrm{CO}_{2}$. The cell medium was changed every 3 days and cells were subcultured at $80 \%$ confluence using $0.25 \%$ trypsin/EDTA solution (Beijing Solarbio Science \& Technology Co., Ltd., Beijing, China).

Colony-forming unit-fibroblast (CFU-F) assay. Assessing the colony forming efficiency of GMSCs, a CFU-F assay was performed. A total of 500 cells of passage 1 were seeded in a $60 \mathrm{~mm}$ petri dish. The culture medium ( $\alpha$-MEM with $10 \%$ FBS) was replaced every 3 days. After 14 days, cells were fixed with $4 \%$ paraformaldehyde at room temperature for $30 \mathrm{~min}$, and stained with $0.1 \%$ crystal violet (Beijing Solarbio Science $\&$ Technology Co., Ltd.) at room temperature for $10 \mathrm{~min}$, then cells were washed with distilled water and dried. The cells counted by an inverted light microscope. A cluster of 50 or more cells was scored as a CFU-F.

Adipogenic differentiation. The forth-passage GMSCs were seeded in 24 -well plates $\left(5 \times 10^{3}\right.$ cells/well) in the $\alpha$-MEM growth medium. Following $24 \mathrm{~h}$, the medium was replaced by adipogenic medium ( $\alpha$-MEM containing $10 \%$ FBS, $0.5 \mathrm{mM}$ IBMX, $200 \mu \mathrm{M}$ indomethacin, $10 \mu \mathrm{M}$ insulin and $10 \mu \mathrm{M}$ dexamethasone; Sigma-Aldrich; Merck KGaA). Cells which cultured in $\alpha$-MEM containing $10 \%$ FBS were as the control group. Cells were stained with Oil Red O (Beijing Solarbio Science \& Technology Co., Ltd.) at room temperature for 10 min to identify the oil globules under light microscopy after two weeks.

Osteogenic differentiation. The forth-passage GMSCs were seeded in 24 -well plates $\left(5 \times 10^{3}\right.$ cells/well) in $\alpha$-MEM growth medium. After $24 \mathrm{~h}$ the culture medium was replaced with osteogenic medium ( $\alpha$-MEM containing 5\% FBS, $50 \mu \mathrm{M}$ ascorbate-2-phosphate, $10 \mathrm{mM} \beta$-glycerophosphate and $0.1 \mu \mathrm{M}$ dexamethasone; Sigma-Aldrich; Merck KGaA). The cells which cultured in $\alpha$-MEM containing 5\% FBS were as the control group. Cells were characterized by Alizarin Red S (Beijing Solarbio Science \& Technology Co., Ltd.) to identify the mineral nodules after 4 weeks.

Flow cytometry. GMSCs $\left(1 \times 10^{6}\right)$ at passage 4 were collected and washed twice by PBS, and then the cells were incubated with monoclonal fluorescein isothiocyanate-conjugated anti-human antibodies for CD45 (cat. no. 368507), CD73 (cat. no. 344015), CD90 (cat. no. 328107) and phycoerythrin-conjugated anti-human antibodies for CD105 (cat. no. 323205) (BioLegend, Inc., San Diego, CA, USA) at $4^{\circ} \mathrm{C}$ for $40 \mathrm{~min}$ in the dark. After the incubation, cells were washed three times and fixed in $1 \%$ paraformaldehyde at room temperature for $30 \mathrm{~min}$. The suspension was analyzed by flow cytometer (Beckman Coulter, Inc., Brea, CA, USA).

GFP transfection of GMSCs. Tracing the fate of GMSCs in mice, the lentiviral vectors with GFP (Shanghai Genechem, Co., Ltd., Shanghai, China) were used to label GMSCs. The first-passage GMSCs $\left(2 \times 10^{3}\right.$ cells/well) were seeded in 6-well plates for $24 \mathrm{~h}$. Then the culture medium was replaced with virus solution diluted by serum-free $\alpha$-MEM. The viral solution was replaced with complete culture medium following transfection for $8 \mathrm{~h}$. The GFP ${ }^{+}$GMSCs were expanded and cells from the third to fifth passage (following $\sim 2$ weeks of culture) were transplanted into animals.

Animal surgery and GMSCs transplantation. After mice were anesthetized by intraperitoneal injection of $10 \%$ chloral hydrate, a 5-0 silk ligature was placed on the two maxillary second molars. The ligature was tied firmly with a triple-knot on the buccal side of the maxillary second molar and left for 4 weeks. The mice were randomly divided into group A and B. Silk ligatures were removed after the maxillary second molars had been tied for 4 weeks and $500 \mu \mathrm{l} \alpha$-MEM containing $1 \times 10^{6}$ GMSCs were injected into Group A mice via the tail vein. Group B mice were injected with $500 \mu \mathrm{l}$ $\alpha$-MEM as the control group. At 1, 2 and 4 weeks post-injection in this study, mice were sacrificed with carbon dioxide and this was confirmed by mice exhibiting a lack of pulse, breathing, corneal reflex and response to firm toe pinch. In addition, peritonitis was not observed in the mice during the experiment.

Tissue preparation and histopathological analysis. The left maxillaries were isolated following perfusion-fixation with $4 \%$ paraformaldehyde at room temperature for $\sim 20 \mathrm{~min}$. The 
tissues were embedded in paraffin following demineralization in $10 \%$ EDTA (Beijing Solarbio Science \& Technology Co., Ltd.) for 4 weeks. Tissue sections, $3-\mu \mathrm{m}$ thick, were cut in the mesial-distal direction. The nearest segments to the central area were selected. Two sections were stained with hematoxylin and eosin (HE; for $5 \mathrm{~min}$ and $30 \mathrm{sec}$, respectively) and Masson trichrome (MT; Weigert's hematoxylin, $10 \mathrm{~min}$; acid ponceau, $5 \mathrm{~min}$; $1 \%$ acetic acid, $1 \mathrm{~min}$ ), respectively, at room temperature. Images were taken under a light microscope at x100 magnification.

Fluorescent microscope observation. To trace the fate of GMSCs in periodontitis mice, fluorescent microscope observation and immunohistochemical staining were performed. The rehydrated sections were washed with PBS for $5 \mathrm{~min}$ and then stained with DAPI (Beijing Solarbio Science $\&$ Technology Co., Ltd.) at room temperature for $5 \mathrm{~min}$. The slices were observed under a fluorescent microscope after washing with PBS.

Immunohistochemical staining for GFP. Immunohistochemical study was performed using a rabbit monoclonal antibody (1:100; cat. no. AB183734; Abcam, Cambridge, USA) against GFP. Sections were treated with $3 \% \mathrm{H}_{2} \mathrm{O}_{2}$ for 10 min to block peroxidase activity following dewaxing and hydrating. Then the sections were incubated with the primary antibody at $37^{\circ} \mathrm{C}$ for $90 \mathrm{~min}$, washed by PBS and incubated with the horseradish peroxidase-conjugated anti-rabbit secondary antibody (PV-6001; 1:10; Origene Technologies, Inc., Beijing, China) at $37^{\circ} \mathrm{C}$ for $30 \mathrm{~min}$. Nuclear staining was performed with hematoxylin at room temperature for $5 \mathrm{~min}$.

Morphometric analysis of alveolar bone loss. The right maxillaries were boiled for $10 \mathrm{~min}$ under 1 bar pressure to remove the soft tissue and then maxillaries were soaked in hydrogen peroxide solution for $12 \mathrm{~h}$. Maxillaries were washed by PBS after staining with methylene blue at room temperature for 10 min. Images of maxillary molar and alveolar were captured under a stereo microscope and assessed by Image-Pro-Plus 6.0 software (Media Cybernetics, Inc., Rockville, MD, USA). Alveolar bone losses were measured as the sum of distances from cementoenamel junction (CEJ) to the alveolar bone crest $(\mathrm{ABC})$ at 6 sites (mesio-palatal cusp, palatal groove, disto-palatal cusp, mesio-buccal cusp, buccal groove and disto-buccal cusp).

Statistical analysis. All data were expressed as the mean \pm standard deviation. Statistical analysis was performed using a statistical package SPSS 19.0, (IBM, Corps., Armonk, NY, USA). Alveolar bone loss levels of the two groups were assessed with an independent-samples t-test. $\mathrm{P}<0.05$ was considered to indicate a statistically significant difference.

\section{Results}

Characterization and transfection of GMSCs. The adherent GMSCs were observed 5-7 d following initiation of the primary culture and reaching $80 \%$ confluence by $14-21 \mathrm{~d}$. Under an optical microscope, the primary GMSCs demonstrated a fibroblast-like spindle shape (Fig. 1A). At $72 \mathrm{~h}$
Table I. Alveolar bone loss levels of the two groups at 1,2 and 4 weeks post-transplantation (mean \pm standard deviation; $\mathrm{mm}, \mathrm{n}=6$ ).

\begin{tabular}{lccc}
\hline Group & $1 \mathrm{~W}$ & $2 \mathrm{~W}$ & $4 \mathrm{~W}$ \\
\hline A & $2.12 \pm 0.59$ & $1.61 \pm 0.18$ & $1.11 \pm 0.25^{\mathrm{a}}$ \\
B & $2.23 \pm 0.33$ & $1.77 \pm 0.27$ & $1.41 \pm 0.15$ \\
\hline
\end{tabular}

${ }^{\mathrm{a}} \mathrm{P}<0.05$ vs. the $\mathrm{B}$ group at $4 \mathrm{~W}$. W, weeks.

post-transfection, stable expression of GFP could be observed under the fluorescence microscope and GMSCs with stable GFP expression were subcultured (Fig. 1B). A large number of colonies $(19 \pm 6 \%)$ were identified following crystal violet staining (Fig. 1C and D). As GMSCs had been cultured in the adipogenic medium for 2 weeks, lipid-rich globules were confirmed by Oil Red $\mathrm{O}$ staining in the cytoplasm of differentiated cells (Fig. 1E). Furthermore, no positive cells were detected in the control group, which was cultured with standard medium (Fig. 1F). When GMSCs had been cultured in the osteogenic medium for 4 weeks, mineralized nodules were observed following Alizarin Red S staining (Fig. 1G). So, no positive cells were detected in the control group, which was cultured with standard medium (Fig. 1H). Flow cytometry analysis revealed that GMSCs were positive for MSCs markers CD73, CD90 and CD105 and expression percentages were $>95 \%$ for all. In addition, GMSCs were negative for hematopoietic cell marker CD45 with an expression percentage $<2 \%$ (Fig. 1I).

Alveolar bone loss determination. The alveolar bone loss was measured as the distance from the CEJ to ABC. At week 1 and 2 following cell transplantation, there were non-significant differences between group A and B. However, alveolar bone loss in group A was significantly decreased compared with group B by the 4th week (Table I; P<0.05; Fig. 2), which indicated promotion of systemically transplanted GMSCs on alveolar bone regeneration.

Histopathological analysis. Histopathological changes of root furcation were observed by $\mathrm{HE}$ and MT staining. At the 1 st week and 2nd-week post-transplantation, inflammatory cell infiltration, deep periodontal pockets, attachment loss and severe alveolar bone destruction was detected in the two groups by HE staining (Fig. 3A). MT staining demonstrated almost all of alveolar bone was blue stained. At the 4th week post-transplantation, alveolar bone loss of group A was significantly decreased compared with group B (Table I). There was more reddish mature stained bone in group $A$ than group B (Fig. 3B).

Homing properties of GMSCs. GFP-positive cells were detected by fluorescence microscopy and immunohistochemical staining which demonstrated that GMSCs can home to periodontal injury sites and promote tissue regeneration. At 1 -week and 2 -weeks post-transplantation, $\mathrm{GFP}^{+}$fibroblast-like cells could be identified in the gingival tissue and 

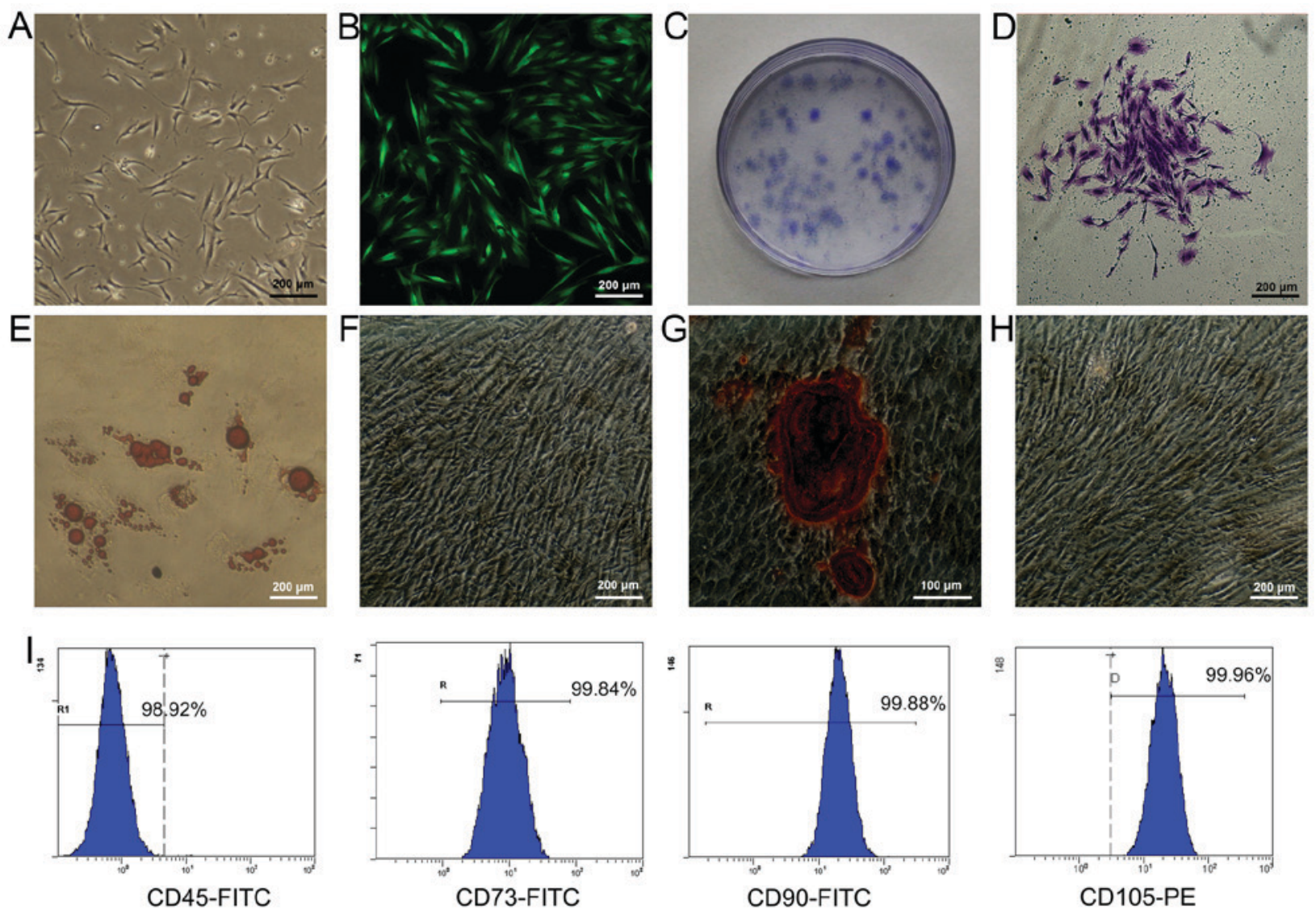

Figure 1. Characterization of GMSCs. (A) Morphology of primary GMSCs. (B) Expression of green fluorescent protein in transfected GMSCs. (C) CFU-F assay at day 14 and colonies were stained with $1 \%$ crystal violet. (D) Cell colonies formed in a CFU-F assay. (E) Lipid droplets were observed after cells were cultured under adipogenic conditions for 2 weeks. (F) Lipid droplets were not detected in the control group following Oil Red O staining. (G) Mineralized nodules were observed after cells were cultured under osteogenic conditions for 4 weeks following Alizarin Red S staining. (H) Mineralized nodules were not detected in the control group following Alizarin Red S staining. Scale bars, $200 \mu \mathrm{m}$. (I) Flow cytometry analysis demonstrated GMSCs were positive for CD73, CD90 and CD105 and negative for CD45. GMSCs, gingival mesenchymal stem cells; CFU-F, colony forming units-fibroblast; FITC, fluorescein isothiocyanate; $\mathrm{PE}$, phycoerythrin.

periodontal ligament. Furthermore, $\mathrm{GFP}^{+}$osteoblasts were identified in the area of newly formed alveolar bone at 4 weeks post-transplantation (Fig. 4).

\section{Discussion}

Gingival tissue is an inherent oral barrier against different insults, including chemicals and bacteria. The most notable characteristics of gingival tissue are its fast reconstitution of tissue and scarless wound healing (15), suggesting that MSCs exist in gingival tissue. Zhang et al (11) first isolated cells possessing MSCs properties within the gingival tissue, these cells displayed a stable phenotype, normal karyotype and telomerase activity and non-tumorigenic in long-term cultures. Compared with other sources of MSCs, GMSCs have a clear advantage, as they are easily obtainable from the discarded tissue in routine dental procedures. Also, there was no significant difference in the characteristics of GMSCs derived from healthy gingival tissue and hyperplastic or inflamed gingival tissue $(16,17)$. In the authors' previous study, it was demonstrated that systemically transplanted GMSCs could home to mandibular defects as other MSCs and promoted bone regeneration involved in novel bone formation. Therefore, it was assumed that systemically transplanted GMSCs could home to periodontal damage sites induced by periodontitis and encourage periodontal tissue regeneration.

To verify the present study's hypothesis, GMSCs were isolated from gingival tissue and the harvested cells displayed self-renewal capability and multilineage differentiation potential in vitro. Furthermore, the results of flow cytometric analysis demonstrated that GMSCs express MSCs markers CD73, CD90 and CD105, but lack CD45. All characteristics above were consistent with the criteria of mesenchymal stromal cells suggested by the International Society for Cellular Therapy (18).

To trace the fate of GMSCs in vivo, the GFP gene was transduced into GMSCs using lentiviral vectors. The lentiviral vectors can integrate exogenous GFP genes into GMSCs chromosomes, therefore achieving persistent expression (19). Most importantly, the integration and expression of GFP genes in mesenchymal stem cells have no significant effect on the characteristics of stem cells. At $72 \mathrm{~h}$ following transfection, $>90 \%$ transfected cells emitted bright green fluorescence under a fluorescent microscope and expressed GFP stably with each passage.

A periodontitis model was established in mice by tying a ligature at the second maxillary molar, then overt bone loss and gingival inflammation were detected at 4 weeks following 
A
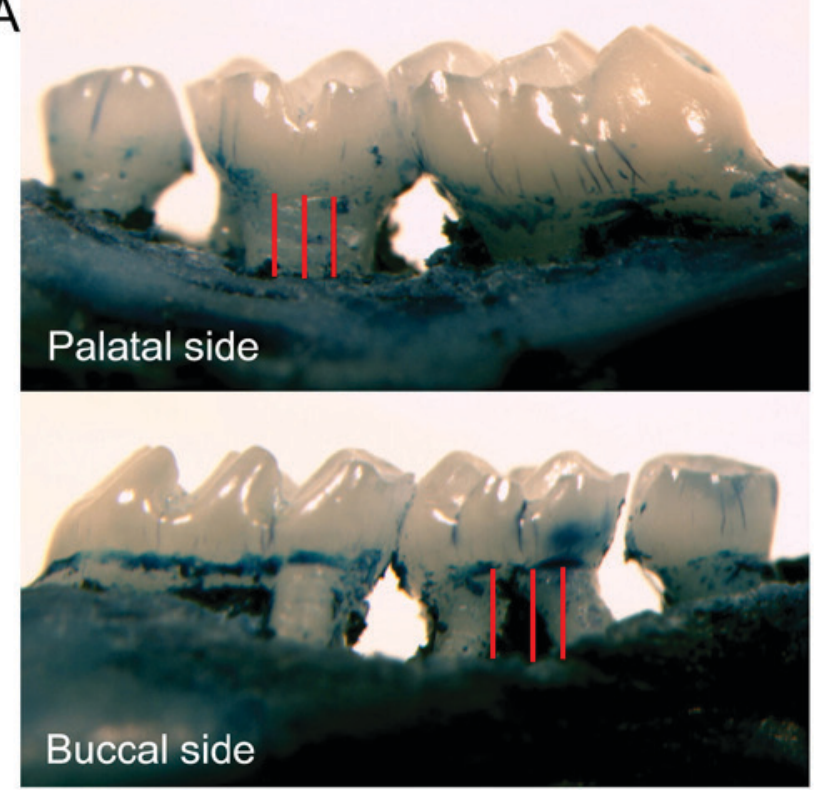

B

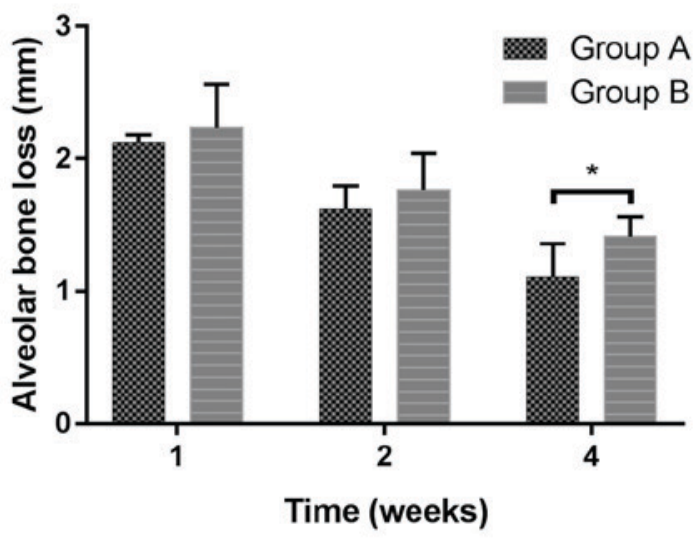

Figure 2. Alveolar bone loss levels. (A) The distance from alveolar bone crest to cementoenamel junction at 6 sites (indicated by red lines) were measured and calculated together as alveolar bone loss levels. (B) The alveolar bone loss level of group B (1.41 $\pm 0.15 \mathrm{~mm})$ was significantly increased compared with group A $(1.11 \pm 0.25 \mathrm{~mm})$ at 4 th week post-transplantation. ${ }^{*} \mathrm{P}<0.05$. There was no statistically significant difference between the A and $\mathrm{B}$ group at the 1 st or $2 \mathrm{nd}$ week post-transplantation.

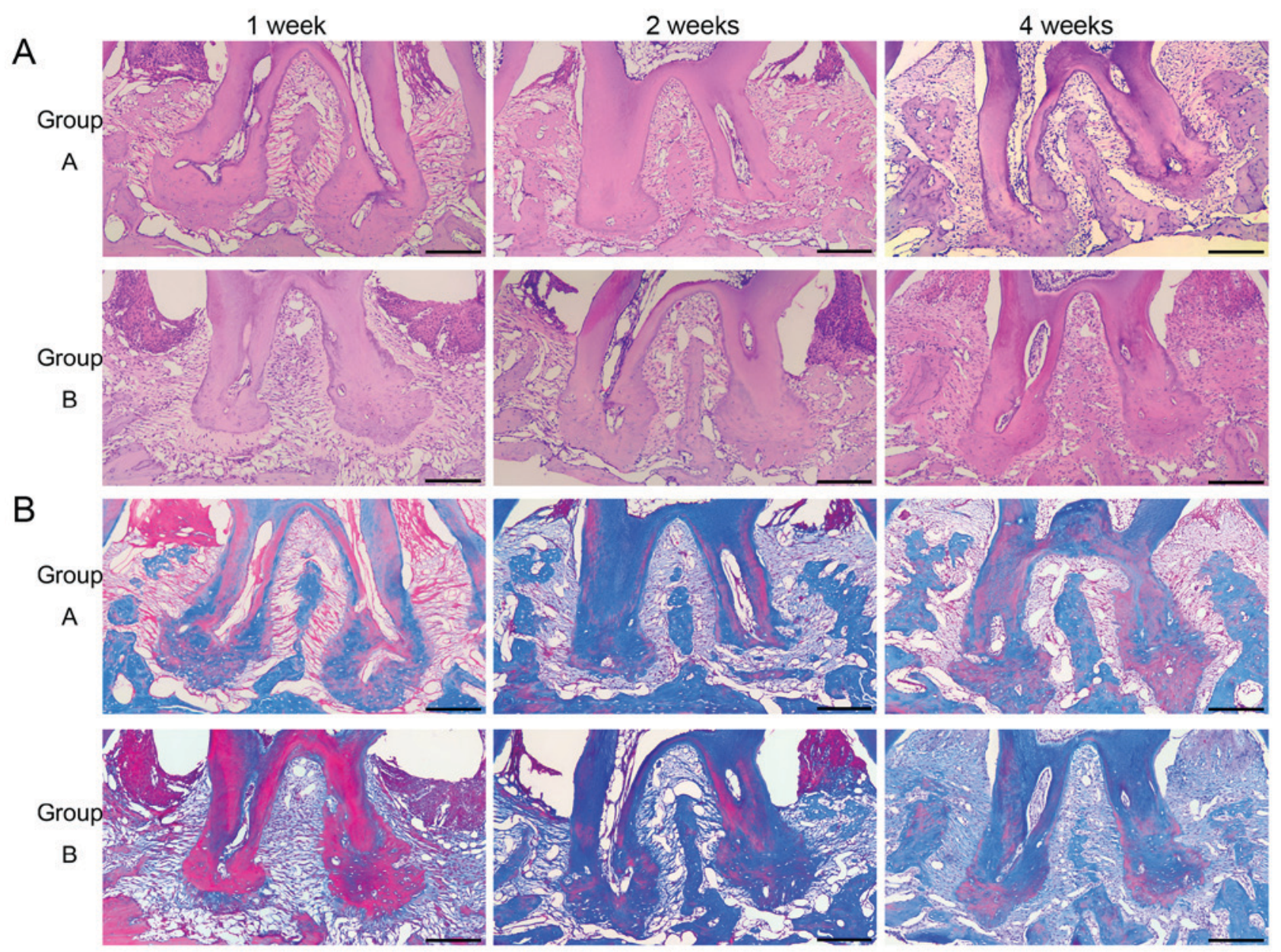

Figure 3. Histopathological changes at root furcation area. (A) Hematoxylin and eosin staining of the root furcation area sections. At the 1st week and 2nd week post-transplantation, severe periodontal inflammation and alveolar bone destruction were detected in the two groups. The alveolar bone heights of group A were significantly increased compared with group B at the 4th week. Magnification: x100. Scale bars, $200 \mu \mathrm{m}$. (B) Masson trichrome staining of the root furcation area sections. Almost all of the alveolar bones were stained blue by the 1st week and 2nd week post-transplantation. At 4th week, more reddish stained mature bone was detected in group A. Magnification: x100. Scale bars, $200 \mu \mathrm{m}$. 

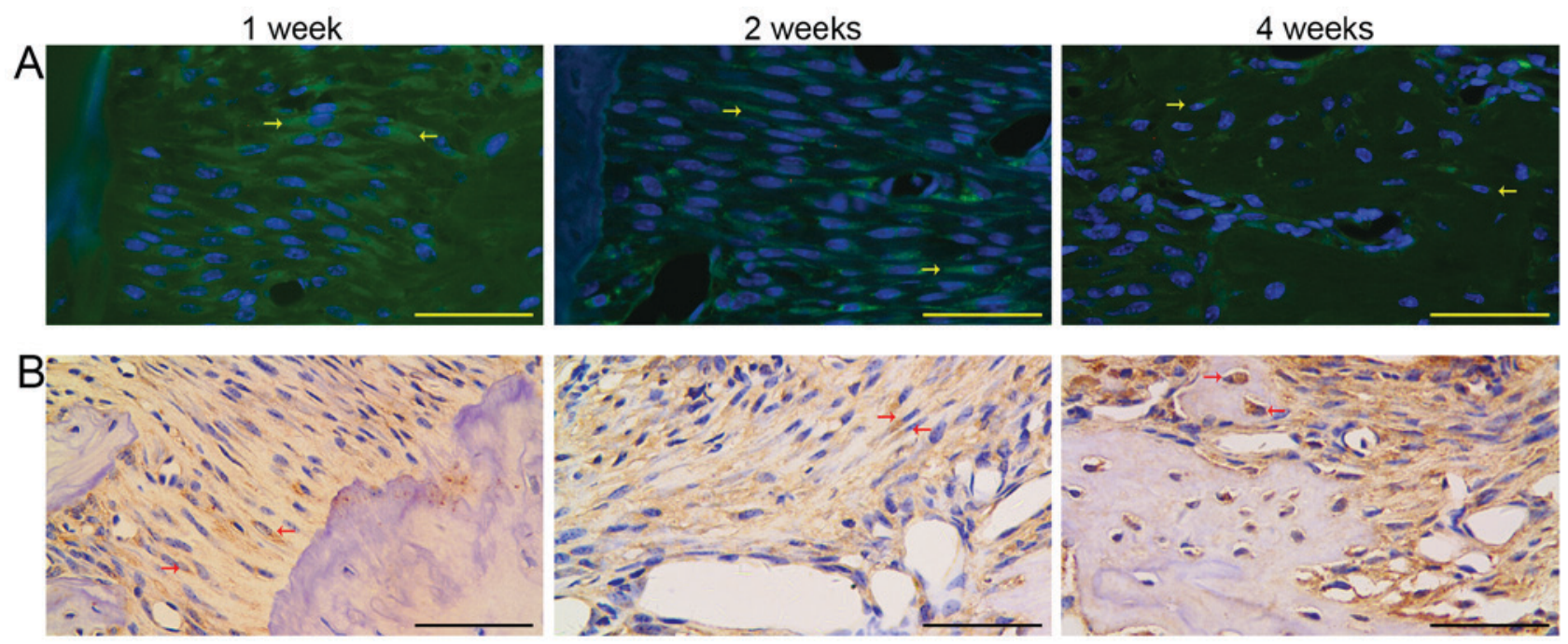

Figure 4. GFP ${ }^{+}$cells in periodontal tissue. (A) Observation under fluorescence microscopy. Cells contained green cytoplasm and blue stained nuclei were detected (as yellow arrowheads indicated). Magnification: x400. Scale bars, $50 \mu \mathrm{m}$. (B) Anti-GFP immunohistochemical staining. GFP gingival fibroblasts, periodontal ligament cells and osteoblasts were detected (as indicated by red arrowheads). Magnification: x400. Scale bars, $50 \mu \mathrm{m}$. GFP, green fluorescent protein.

ligation. A total of 12 sites on alveolar bone were susceptible to bone loss following ligature-induced periodontitis in mice (20). In this study, the alveolar bone loss was measured as the sum of distances from the CEJ to the ABC at 6 sensitive sites of the second maxillary molars. As a result, alveolar bone heights in group A were increased compared with group B at 4 weeks post-transplantation. Results of HE and MT staining demonstrated more newly formed bone and higher alveolar bone heights in group A at 4 weeks post-transplantation. These results proved that GMSCs are effective in promoting bone regeneration. In addition, compared with group B, reduced inflammatory cell infiltration and more periodontal attachment were demonstrated in group A at 2 and 4 weeks post-transplantation. This suggests that GMSCs have an anti-inflammatory effect on periodontitis, but the specific anti-inflammatory mechanism should be a key task in future research.

Fluorescent microscopy and anti-GFP immunohistochemical staining were performed to trace the fate of GFP-labeled GMSCs in vivo. $\mathrm{GFP}^{+}$gingival fibroblasts and periodontal ligament cells were detected at 1 and 2 weeks post-transplantation. In addition, $\mathrm{GFP}^{+}$osteoblasts can be detected in the newly formed bone area by the 4 th-week post-transplantation. The results proved that GMSCs could home to periodontal defect sites and differentiate into periodontal tissue cells. Previous studies have demonstrated that the microenvironment of MSCs can induce the transplanted cells to differentiate into specific tissues (21-23). At the same time, transplanted stem cells also produce bioactive molecules which stimulate the precursor cells to differentiate (24). The question of whether the same mechanism occurs in GMSCs and periodontitis remains to be answered. Periodontal disease is a chronic disease developing with an active stage and resting stage appearing alternately. The model established in this study simulates an acute episode of periodontitis. Future studies should put more effort into clarifying the therapeutic effect and mechanism of GMSCs on chronic inflammation.
In conclusion, GMSCs have been identified that possess characteristics of MSCs and GMSCs could home to the inflammatory periodontium sites and be employed in novel tissue formation. GMSCs serve an essential anti-inflammation role, although the exact mechanism is unclear so far.

\section{Acknowledgements}

The authors would like to thank The Key Lab of Oral Clinical Medicine and The Central Lab of Affiliated Hospital of Qingdao University for the use of laboratories.

\section{Funding}

The present study was supported by grants from the National Natural Science Foundation of China (grant no. 81500849) to Dr Quanchen Xu and Shandong Province Key Research Plan (grant no. 2018GSF118150) to Dr Quanchen Xu.

\section{Availability of data and materials}

The datasets used and/or analyzed during the present study are available from the corresponding author on reasonable request.

\section{Authors' contributions}

ZW, QX and HS designed the study. WS, RH, XL and JY performed the experiments. QX analyzed the data and WS wrote the manuscript. All authors discussed the results and reviewed the manuscript.

\section{Ethics approval and consent to participate}

Ethical approval for the study was granted from the Affiliated Hospital of Qingdao University (Qingdao, China) and informed consent was provided. 


\section{Patient consent for publication}

Not applicable.

\section{Competing interests}

The authors declare that they have no competing interests.

\section{References}

1. Kassebaum NJ, Bernabé E, Dahiya M, Bhandari B, Murray CJ and Marcenes W: Global burden of severe periodontitis in 1990-2010: A systematic review and meta-regression. J Dent Res 93 : 1045-1053, 2014.

2. Hynes K, Menicanin D, Gronthos S and Bartold PM: Clinical utility of stem cells for periodontal regeneration. Periodontology 59: 203-227, 2012

3. Salvi GE, Mischler DC, Schmidlin K, Matuliene G, Pjetursson BE, Brägger U and Lang NP: Risk factors associated with the longevity of multi-rooted teeth. Long-term outcomes after active and supportive periodontal therapy. J Clin Periodontol 41: 701-707, 2014.

4. Silvério KG, Benatti BB, Casati MZ, Sallum EA and Nociti FH Jr: Stem cells: Potential therapeutics for periodontal regeneration. Stem Cell Rev 4: 13-19, 2008.

5. Luria EA, Panasyuk AF and Friedenstein AY: Fibroblast colony formation from monolayer cultures of blood cells. Transfusion 11: 345-349, 1971

6. Du J, Shan Z, Ma P, Wang S and Fan Z: Allogeneic bone marrow mesenchymal stem cell transplantation for periodontal regeneration. J Dent Res 93: 183-188, 2014.

7. Seo BM, Miura M, Gronthos S, Bartold PM, Batouli S, Brahim J, Young M, Robey PG, Wang CY and Shi S: Investigation of multipotent postnatal stem cells from human periodontal ligament. Lancet 364: 149-155, 2004.

8. Huang TJ, Sonoyama W, Chen J and Sang HP: In vitro characterization of human dental pulp cells: Various isolation methods and culturing environments. Cell Tissue Res 324: 225-236, 2006

9. Morsczeck C, Götz W, Schierholz J, Zeilhofer F, Kühn U, Möhl C, Sippel C and Hoffmann KH: Isolation of precursor cells (PCs) from human dental follicle of wisdom teeth. Matrix Biol 24: 155-165, 2005.

10. Tomar GB, Srivastava RK, Gupta N, Barhanpurkar AP, Pote ST, Jhaveri HM, Mishra GC and Wani MR: Human gingiva-derived mesenchymal stem cells are superior to bone marrow-derived mesenchymal stem cells for cell therapy in regenerative medicine. Biochem Biophys Res Commun 393: 377-383, 2010.

11. Zhang Q, Shi S, Liu Y, Uyanne J, Shi Y, Shi S and Le AD: Mesenchymal stem cells derived from human gingiva are capable of immunomodulatory functions and ameliorate inflammation-related tissue destruction in experimental colitis. J Immunol 183: 7787-7798, 2009.
12. Su WR, Zhang QZ, Shi SH, Nguyen AL and Le AD: Human gingiva-derived mesenchymal stromal cells attenuate contact hypersensitivity via prostaglandin E2-dependent mechanisms. Stem Cells 29: 1849-1860, 2011.

13. Wada N, Wang B, Lin NH, Laslett AL, Gronthos S and Bartold PM: Induced pluripotent stem cell lines derived from human gingival fibroblasts and periodontal ligament fibroblasts. J Periodontal Res 46: 438-447, 2011.

14. Xu QC, Wang ZG, Ji QX, Yu XB, Xu XY, Yuan CQ, Deng J and Yang PS: Systemically transplanted human gingiva-derived mesenchymal stem cells contributing to bone tissue regeneration. Int J Clin Exp Pathol 7: 4922-4929, 2014.

15. Häkkinen L, Uitto VJ and Larjava $\mathrm{H}$ : Cell biology of gingival wound healing. Periodontology 24: 127-152, 2000.

16. Ge S, Mrozik KM, Menicanin D, Gronthos S and Bartold PM: Isolation and characterization of mesenchymal stem cell-like cells from healthy and inflamed gingival tissue: Potential use for clinical therapy. Regen Med 7: 819-832, 2012.

17. Tang L, Li N, Xie H and Jin Y: Characterization of mesenchymal stem cells from human normal and hyperplastic gingiva. J Cell Physiol 226: 832-842, 2011.

18. Dominici M, Le Blanc K, Mueller I, Slaper-Cortenbach I, Marini F, Krause D, Deans R, Keating A, Prockop Dj and Horwitz E: Minimal criteria for defining multipotent mesenchymal stromal cells. The International Society for Cellular Therapy position statement. Cytotherapy 8: 315-317, 2006.

19. Ye Z, Yu X and Cheng L: Lentiviral gene transduction of mouse and human stem cells. Methods Mol Biol 430: 243-253, 2008.

20. Abe T and Hajishengallis G: Optimization of the ligature-induced periodontitis model in mice. J Immunol Methods 394: 49-54, 2013.

21. Liechty KW, Mackenzie TC, Shaaban AF, Radu A, Moseley AM, Deans R, Marshak DR and Flake AW: Human mesenchymal stem cells engraft and demonstrate site-specific differentiation after in utero transplantation in sheep. Nat Med 6: 1282-1286, 2000.

22. Guo X, Bai Y, Zhang L, Zhang B, Zagidullin N, Carvalho K, $\mathrm{Du} \mathrm{Z}$ and Cai B: Cardiomyocyte differentiation of mesenchymal stem cells from bone marrow: New regulators and its implications. Stem Cell Res Ther 9: 44, 2018.

23. Aggarwal S and Pittenger MF: Human mesenchymal stem cells modulate allogeneic immune cell responses. Blood 105: 1815-1822, 2005.

24. Seo BM, Miura M, Sonoyama W, Coppe C, Stanyon R and Shi S: Recovery of stem cells from cryopreserved periodontal ligament. J Dent Res 84: 907-912, 2005.

(i) $\ominus$ This work is licensed under a Creative Commons Attribution-NonCommercial-NoDerivatives 4.0 International (CC BY-NC-ND 4.0) License. 